Tohoku J. Exp. Med., 2011, 223, 113-118

\title{
A Novel PAX4 Mutation in a Japanese Patient with Maturity- Onset Diabetes of the Young
}

\author{
Wakako Jo, ${ }^{1}$ Machiko Endo, ${ }^{2}$ Katura Ishizu, ${ }^{1}$ Akie Nakamura ${ }^{1}$ and \\ Toshihiro Tajima ${ }^{1}$
}

\author{
${ }^{1}$ Department of Pediatrics, Hokkaido University School of Medicine, Sapporo, Japan \\ ${ }^{2}$ Department of Pediatrics, Hakodate Goryokaku Hospital, Hakodate, Japan
}

\begin{abstract}
Maturity-onset diabetes of the young (MODY) is a genetically and clinically heterogeneous type of diabetes mellitus, characterized by early onset (often before 25 years of age) and absence of pancreatic autoimmunity markers. Paired-homeodomain transcription factor 4 (PAX4) functions as a transcriptional repressor and is involved in the differentiation of insulin-secreting $\beta$-cells. Here we identified a novel PAX4 mutation in a Japanese patient with MODY. A 15-year-old, non-obese boy was admitted to our hospital because of polyuria and polydipsia. Laboratory evaluation showed an elevated fasting glucose level; however, islet cell antibodies and glutamic acid decarboxylase antibodies were not detected in the patient's serum. The proband's father had been diagnosed as having type 2 diabetes at age of 30 years. We therefore analyzed several candidate genes of MODY, and identified a novel mutation of a 39-base heterozygous deletion in exon 3 (c.374-412 del39) of PAX4 in the proband and his father. This mutation may cause exon 3 skipping that results in a frameshift, thereby producing a premature stop codon in exon 5. As this mutant PAX4 lacks a part of the homeodomain that is critical for binding to the target gene, this mutant was thought to lose the transcriptional repressor function. As expected, luciferase-reporter assays revealed that the mutant PAX4 could not repress the activities of insulin and glucagon gene promoters, unlike the wild-type PAX4 that repressed the promoter activities. The present study demonstrates that a novel mutation of PAX4 is likely to be associated with diabetes in this Japanese family.
\end{abstract}

Keywords: Maturity onset of diabetes of young (MODY); PAX4; mutation

Tohoku J. Exp. Med., 2011, 223 (2), 113-118. (C) 2011 Tohoku University Medical Press

Maturity-onset diabetes of the young (MODY) is a genetically and clinically heterogeneous type of diabetes mellitus characterized by early onset (often before 25 years of age) and absence of pancreatic autoimmunity markers (Fajans et al. 2001). Mutations of six distinct genes have been implicated in the six different types of diabetes (MODY1-6). One of the six genes encodes the glycolytic enzyme glucokinase (GCK) (associated with MODY2) and the other five encode transcription factors: hepatocyte nuclear factor (HNF4A) (MODY1), HNF1A (MODY3), insulin promoter factor 1 (IPF1) (MODY4), HNF1B (MODY5) and NEUROD1 (MODY6) (Malecki et al. 1992; Vionnet et al. 1992; Yamagata et al. 1996a, 1996b; Horikawa et al. 1997; Stoffers et at. 1997). However, as genetic defects of these genes are not frequently seen among Japanese MODY patients (Nishigori et al. 1993; Furuta et al. 2002; Kagami-Takasugi et al. 2006), mutations in other genes may be responsible for the development of MODY.

The paired homeodomain transcriptional factor, PAX4, functions as a transcriptional repressor through a paired domain and homeodomain (Sosa-Pineda et al. 1997; Smith et al. 1999; Sosa-Pineda 2004; Brun and Gauthier 2008). In heterozygous Pax 4 knockout mice, mature $\beta$-cell and $\delta$ cells did not differentiate and proliferate, and thus PAX4 is thought to be essential for the commitment of progenitor cells to the different islet cell lineages. In addition, PAX4 is likely to be important for regeneration of $\beta$-cells in adult life (Brun and Gauthier 2008).

It was reported that a $P A X 4$ mutation was associated with type 2 diabetes (Shimajiri et al. 2001). Thereafter, two possible pathogenic mutations of PAX4 in two Thai patients with MODY were described (Plengvidhya et al. 2007). These findings suggest that PAX4 mutation may be responsible for MODY.

Here we analyzed PAX4 in a Japanese family with MODY, and have identified a novel 39-base deletion mutation (c.374-412 del39).

Received October 26, 2010; revision accepted for publication January 11, 2011. doi: 10.1620/tjem.223.113

Correspondence: Toshihiro Tajima, M.D., Ph.D., Department of Pediatrics, Department of Pediatrics, Hokkaido University School of Medicine, N15, W7, Sapporo 060-0835, Japan.

e-mail: tajeari@med.hokudai.ac.jp 


\section{Clinical report}

A 15-year-old, non-obese boy was admitted to our hospital because of polyuria and polydipsia. On physical examination, his height was $161.5 \mathrm{~cm}$ and body weight was $47.2 \mathrm{~kg}$. His blood pressure was $115 / 67 \mathrm{mmHg}$. Laboratory evaluation showed that the fasting glucose level was $642 \mathrm{mg} / \mathrm{dl}$ and urine ketone bodies were positive. Ketoacidosis was not observed (pH of venous blood 7.36). Other biochemical findings were within normal limits. The HbA1c level was $14.5 \%$ (NGSP) (Seino et al. 2010). Islet cell antibodies (ICAs) and glutamic acid decarboxylase (GAD) antibodies were not detected in the serum. Urinary C-peptide concentrations measured on two different days were lower than normal range $(13.1 \mu \mathrm{g} /$ day and $18.8 \mu \mathrm{g} /$ day, normal range $20.1 \sim 155 \mu \mathrm{g}$ /day). Blood C-peptide levels (fasting /postprandial) were also low $(1.08 / 1.25 \mathrm{ng} / \mathrm{ml})$ despite the elevated blood glucose levels $(244 / 342 \mathrm{ng} / \mathrm{ml})$. Abdominal computed tomography revealed a normal-sized pancreas and kidneys. Since his capacity of insulin secretion was thought to be decreased, insulin therapy was started at this time. His most recent insulin requirement and $\mathrm{HbA} 1 \mathrm{c}$ level were 0.5 unit $/ \mathrm{kg}$ and $7.4 \%$ (NGSP), respectively. The proband's father had also been diagnosed as having type 2 diabetes at age $30 \mathrm{yr}$. Data on the father at the time that he had been diagnosed, were not available. At this time, the father's weight was $85 \mathrm{~kg}$ and his BMI was $32 \mathrm{~kg} / \mathrm{m}^{2}$. The proband's father's diabetes has been well controlled by only diet therapy without insulin administration.

\section{Methods}

\section{DNA amplification and sequence analysis}

The ethical committee of Hokkaido University School of Medicine approved this study. Written informed consent to participate in the study was obtained from the patient's parents and the patient. Genomic DNA was extracted from peripheral leukocytes. Each exon and exon-intron boundaries of HNF4A, HNF1A, IPF1, HNF1B, NEUROD1 GCK and PAX4 were amplified by polymerase chain reaction (PCR). After amplification, the PCR products were purified and sequenced directly using an ABI PRISM Dye Terminator Cycle Sequencing Kit and an ABI 373A automated florescent sequencer (PE Applied Biosystems, Foster City, CA). Primers for the PCR reactions are shown in Table 1.

$m R N A$ analysis

In order to analyze the effect of the mutation on mRNA expression, a mini-gene construct was produced. The normal and mutant alleles of the PAX4 gene from exon 1 to exon 4 were amplified from the proband's genomic DNA with a set of primers 5'-ATGAACCAG CTTGGGGGG-3' in exon 1 (codon 1 to codon 6 in exon 1, primer 1) and 5'-CAGTGCCTCTGCTTGGCT-3' in exon 4 (codon 177 to codon 172, primer 2). Thereafter, the PCR products were cloned into the pCR 3.1 TA cloning vector (Invitrogen, Carlsbad, CA). We selected several clones and verified the DNA sequence of the normal and mutant alleles. Each of the normal and mutant alleles was cloned into the expression vector, pCDNA3 (Invitrogen, Carlsbad CA), using the appropriate restriction enzyme. The normal and mutant constructs were designated as WT-gPAX4 and MT-gPAX4, respectively.

In order to analyze the mRNA expression of WT-gPAX4 and MT-gPAX4, COS7 (African green monkey SV40-transformed kidney fibroblast) and MIN6 cells (immortalized insulin-secreting pancreatic $\beta$ cells) were transiently transfected using Lipofectamine 2000 (Promega, Madison, MI) with either WT-gPAX4 (1.0 $\mu \mathrm{g})$ or MTgPAX4 $(1.0 \mu \mathrm{g})$. Total RNA extraction and RT-PCR were done using FastPure RNA kit and PrimeScript RT-PCR kit (Takara, Tokyo, Japan). The obtained cDNA from WT-gPAX4 or MT-gPAX4 was purified and sequenced directly.

Wild-type and mutant PAX4 cDNA construction and plasmid construction

Human PAX4 cDNA was amplified from PCR Ready First Strand DNA of normal human placenta (BioChain Institute, Inc) by PCR and subcloned into a pcDNA 3.1 expression vector. The mutant PAX4-cDNA was created by the PrimeSTAR mutagenesis kit (Takara, Tokyo, Japan). The wild and mutant PAX4 cDNAs were designated as WT-cPAX4 and MT-cPAX4, respectively.

The human insulin and glucagon promoters were amplified by PCR and subcloned into pGL3 reporter vectors (Promega, Madison, $\mathrm{MI}$ ) and were designated as pGL3-insulin and pGL3-glucagon, respectively.

Luciferase assay

MIN6 cells were obtained from American Type Cell Culture (Manassas, VA) and grown in Dulbecco's modified Eagle's medium (DMEM) supplemented with $15 \%$ fetal bovine serum.

Table 1. Sequences of primers used to amplify PAX4.

\begin{tabular}{cll}
\hline Exon & \multicolumn{1}{c}{ Forward $\left(5^{\prime}-3^{\prime}\right)$} & \multicolumn{1}{c}{ Reverse $\left(5^{\prime}-3^{\prime}\right)$} \\
\hline 1 & AGGTGGTGTTGGATACCTC & CAGGCTCTTGCCTTCAGAG \\
2 & CCATCATGCCTCACCTGTC & GCCTCTTTTCCAGCCCCAGT \\
3 & CCTGAGTCTGAGCACCATCTC & GATTTGGCTGTGATTAGCCC \\
4 & CTGACCAGAGGAATCACCATC & CCCTGTGTCACACTGAGGAC \\
5 & GAGACCCATGCCTTGCTCCTC & GGCCCAGACTCTTCCTCCTTG \\
6 & GATCAGCAGGTGACAGGCAGC & GATGACTGAGCGGGCAGATG \\
7 & AGTGGCTGACTTTCCTAGAAC & GAGCCCATGAGCCCTTCAGTC \\
8 & CTCTACAGGAGGCATCACTG & GAGGTTGAGTCAGTCGACCCT \\
9 & GTCCCCACAGGCCACTTGCC & TGGGCAGGATGGTATTAGATCT \\
\hline
\end{tabular}


In order to assay insulin and glucagon gene promoter activity, MIN6 cells were plated in 6-well plates to $70 \%$ confluence and transiently transfected using Lipofectamine 2000 with the empty expression vector $(1.0 \mu \mathrm{g})$, WT-cPAX4 $(1.0 \mu \mathrm{g})$, MT-cPAX4 $(1.0 \mu \mathrm{g})$, or WT-cPAX4 $(0.5 \mu \mathrm{g})$ plus MT-cPAX4 $(0.5 \mu \mathrm{g})$ together with each reporter vector $(0.5 \mu \mathrm{g})$ (pGL3-insulin or pGL3-glucagon) according to the previous report (Plengvidhya et al. 2007). Cell extracts were prepared 48 hours after transfection and luciferase assays were performed. Luciferase measurements were divided by $\beta$-galactosidase activity to control for transfection efficiency. Transfections were performed in triplicate within a single experiment, and the experiment was repeated three times. The mean of each triplicate was expressed as a percentage of the empty vector control to allow comparison of data from different experiments. The significance of differences between the wild type and mutant was assessed by one-way ANOVA followed by Scheffer's post hoc test. A $P$-value of $<0.05$ was considered significant.

\section{Fluorescence analysis and microscopy}

COS7 cells transfected with either WT-PAX4-GFP or MT-PAX4-GFP were placed onto glass coverslips and fixed in $4 \%$ (vol/vol) formaldehyde/PBS $24 \mathrm{hr}$ after transfection. Cells were permeabilized in $0.1 \%$ Triton/PBS and then examined under a Fuji fluorescence microscope (Fuji, Tokyo, Japan).

\section{Results}

\section{Gene sequencing results}

Sequence analysis of genomic PAX4 demonstrated a heterozygous 39-base deletion mutation in exon 3 (c.374412 del39) (Fig. 1A). His father also had this heterozygous mutation. One hundred fifty normal Japanese subjects did not have this deletion.

\section{$m R N A$ analysis}

RT-PCR analysis after transfection of WT-gPAX4 demonstrated a $531 \mathrm{bp}$ cDNA as expected, whereas RT-PCR analysis of MT-gPAX4 yielded a shorter cDNA fragment (Fig. 2A) in COS7 cells. Sequence analysis of this shorter cDNA fragment demonstrated the lack of exon 3 (Fig. 2B). The exon 3 skipping changed the open reading frame, resulting in a premature stop codon at codon 206 in exon 5. The same results were obtained when using MIN6 cells.

\section{Functional analysis}

Cotransfection of WT-cPAX4 with insulin and glucagon promoters repressed the luciferase reporter gene relative to the empty vector; however, MT-cPAX4 lacked the repressor activity, indicating loss-of-function (Fig. 3A, B). Co-transfection of MT-cPAX4 and an equivalent amount of WT-cPAX4 did not affect the activity of WT-cPAX4, which

(A)

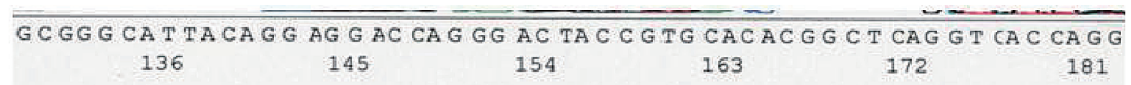

WT

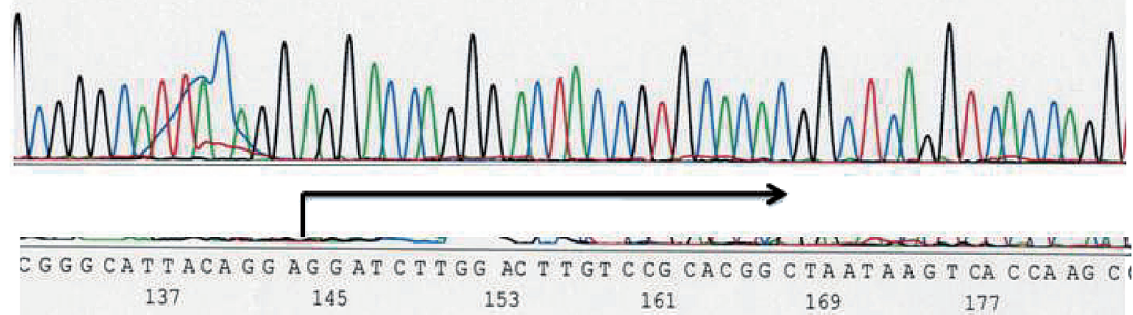

MT

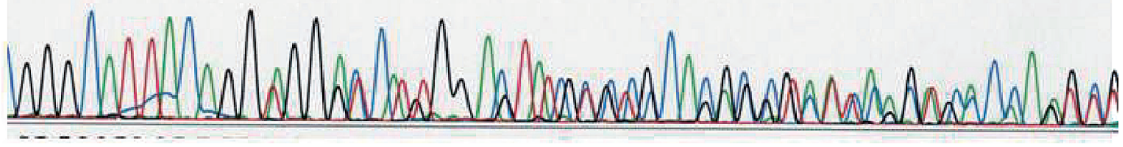

39-base heterozy gous deletion premature stop codon

(B)

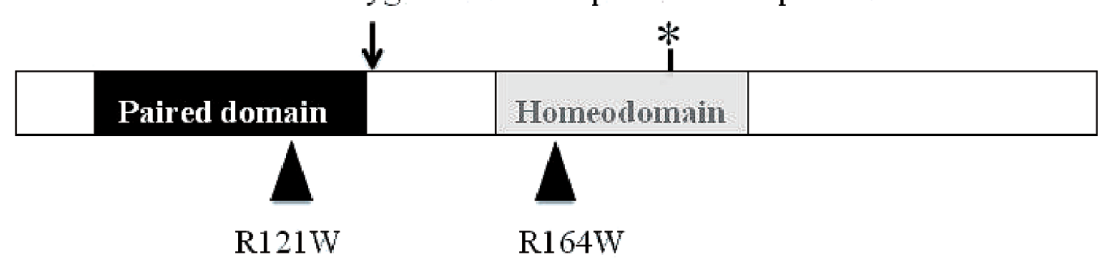

Fig. 1. Sequence analysis of $P A X 4$.

(A) Sequence analysis of genomic DNA from the patient. Sequence analysis of the PAX4 gene demonstrated a 39-base deletion (c. 374-412 del39). Note that after mutation, double bands are present (arrow). (B) Schematic representation of human the PAX4 protein. The black box represents paired domain and the shaded box represents homeodomain. Two previously-reported pathogenic missense mutations (arrowheads) and the mutation found in this study (arrow) are displayed. The deletion mutation found in the present study produces a premature stop codon at codon 206 (asterisk) in the homeodomain. 
(A)

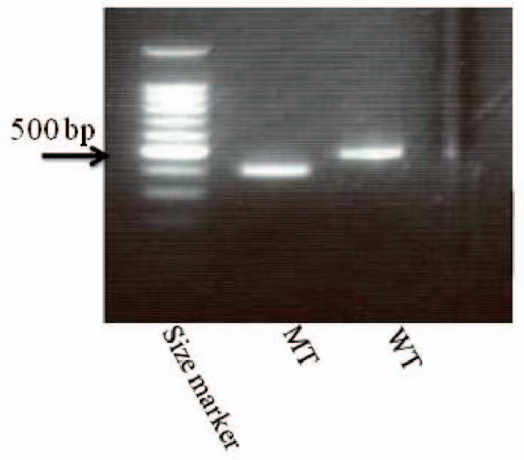

(B)

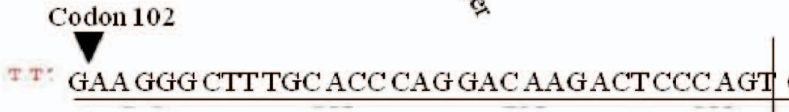

\section{WT}
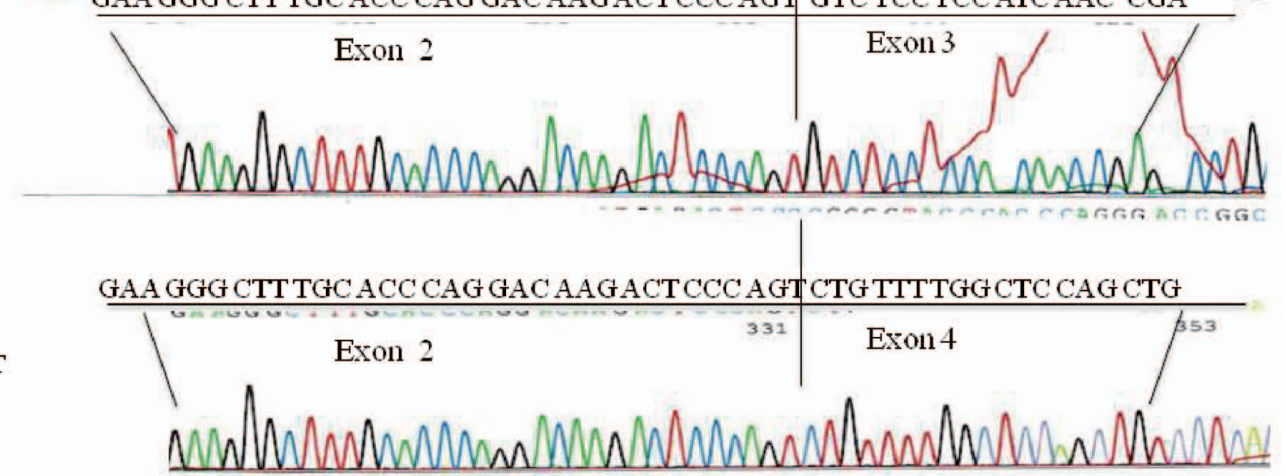

Fig. 2. RT-PCR analysis of the mutation.

(A) RT-PCR analysis of PAX4 after transfection of WT-gPAX4 demonstrated a 531 bp cDNA as expected, whereas RT-PCR analysis after transfection of MT-gPAX4 yielded a shorter cDNA fragment. (B) Sequence analysis of this shorter cDNA demonstrated the lack of exon 3. The sequence of the cDNA from WT-gPAX4 is also shown.

excluded dominant negative effects (Fig. 3A, B).

Next, we analyzed the subcellular localization of WT-cPAX4 and MT-cPAX4. Both WT-cPAX4 and MT-cPAX4 proteins were localized to the nucleus (Fig. 3C).

\section{Discussion}

We identified a novel heterozygous 39-base deletion in the PAX4 gene in a Japanese family with MODY. The authentic splice site of exon 3-intron 3 was still present in the mutant sequence; however, RT-PCR analysis demonstrated that this mutation led to exon 3 skipping and changed the open reading frame, resulting in a premature stop codon at codon 206 in exon 5. It is considered that exonic-cis elements, distinct from the classical splicing signals, are important for correct splice-site identification. These elements work as exonic splicing enhancers (ESEs) and are necessary for regulation of alternative splicing (Blencowe 2000). In silico analysis of ESE motifs in the wild type and mutant exon 3 revealed that the 39-base deleted region in exon 3 is located on several ESE sequence motifs and was predicted to influence ESEs (Table 2). Thus, this deletion probably disrupts these recognition sites such that exon inclusion may no longer be favored, resulting in exon skipping and the formation of aberrant transcripts. Indeed, several mutations of ESE sequences have been reported to cause exon skipping in human diseases (Moseley et al. 2002; Levit et al. 2010).
Our in vitro study revealed that this mutant PAX4 had reduced repression activity toward target genes compared with the wild type. The original papers demonstrated that PAX families' functions as a transcription repressor through a paired domain and homeodomain (Fujitani et al. 1999; Smith et al. 1999). As our mutant lacked the homeodomain, our in vitro results were consistent with these previous notions. Furthermore, our mutant did not demonstrate a dominant negative effect similar to the previously reported R121W and R164W mutations (Shimajiri et al.2001; Plengvidhya et al. 2007). In vivo, the functional alteration induced by the deletion could be due to nonsensemediated decay (NMD) (Rehwinkel et al. 2006). Degradation of mutant PAX4 via the NMD mechanism could prevent translation of the aberrant transcripts by eliminating mRNAs that contain a premature stop codon. Thus, the haploinsufficiency or reduced gene dosage of PAX4 may have led to the development of diabetes in our family. However, it is noted that heterozygous Pax4 knockout mice did not develop diabetes (Brun and Gauthier 2008). This may imply that humans are more sensitive than mice to the dosage of PAX4.

In the family in our study, the phenotypes of the proband and his father were different. Despite having the identical genotype of PAX4, the diabetes in the father has been controlled well, while his son requires insulin therapy. At the time of diagnosis of diabetes, the father had been obese, 

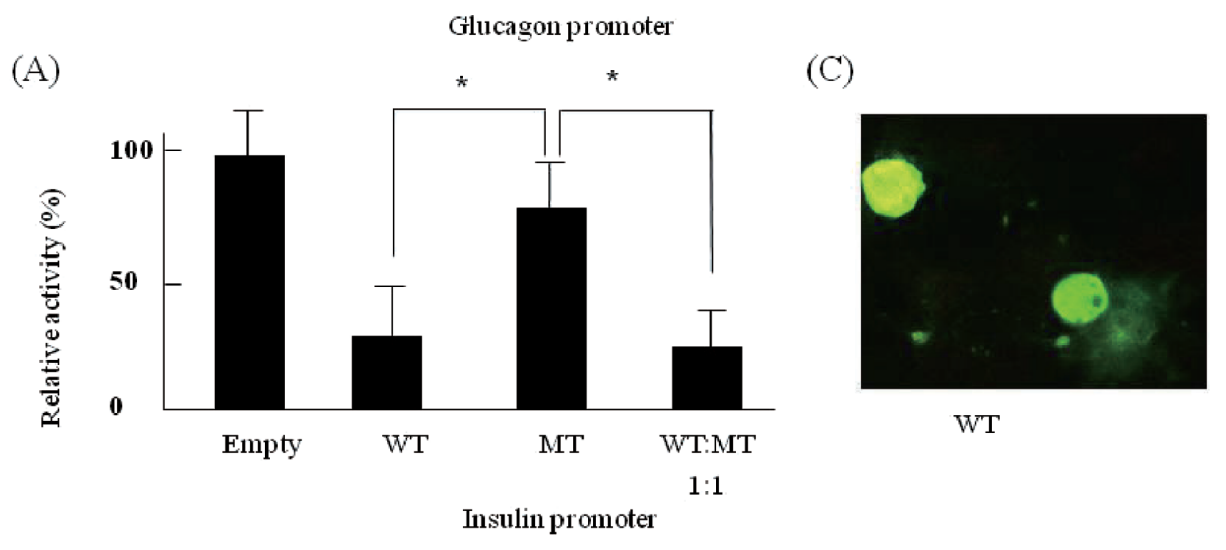

WT

(B)

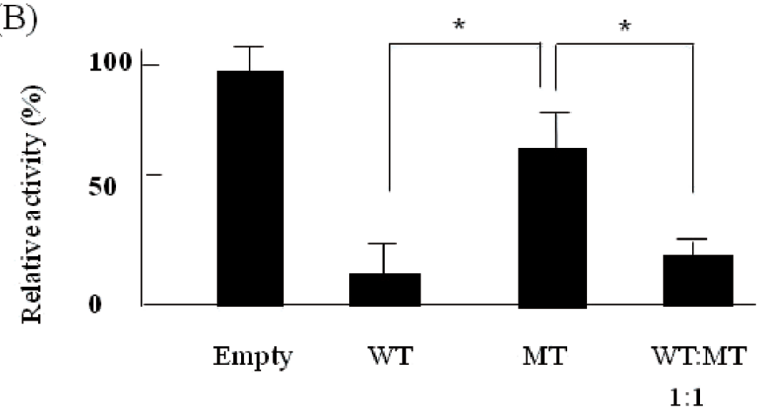

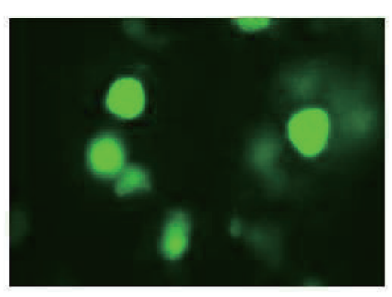

MT

Fig. 3. In vitro functional analysis of WT and MT-PAX4.

(A) WT-cPAX4 represses glucagon (A) and insulin (B) promoter activity, but MT-cPAX4 has reduced suppressive activity. Co-transfection of MT-cPAX4 and an equivalent amount of WT-cPAX4 does not affect the function of WT-cPAX4, suggesting no dominant negative effect. Data are expressed as mean \pm S.D. $(n=6)$ from the analysis for three times. $*, P<0.01$.

(C) Subcellular localization of WT-PAX4 and MT-PAX4. COS7 cells expressing WT-PAX4-GFP or MT-PAX4-GFP were visualized directly by GFP fluorescence. Both WT-PAX4 and MT-PAX4 are localized at the nucleus.

Table 2. Predictions of exonic splice enhancers.

\begin{tabular}{|c|c|c|c|c|c|}
\hline \multicolumn{3}{|c|}{ WT-Exon 3} & \multicolumn{3}{|c|}{ MT-Exon 3} \\
\hline Motif & Site & Score & & Site & Score \\
\hline SRp40 & ttacagg & 6.01235 & & ttacagg & 6.01235 \\
\hline SRp55 & cacggc & 3.88260 & Deleted* & tacagg & 2.34137 \\
\hline SF2/ASF & cacacgg & 5.86231 & Deleted* & attacag & -2.44998 \\
\hline $\begin{array}{l}\text { SF2/ASF } \\
\text { (IgM-BRCA1) }\end{array}$ & cacacgg & 4.96554 & Deleted* & attacag & -2.65315 \\
\hline $\mathrm{SC} 35$ & gactaccg & 4.74311 & Deleted* & attacagg & -2.81196 \\
\hline
\end{tabular}

*By the 39 bases deletion, MT-Exon 3 lacked these motifs.

but we did not have data on his insulin secretion. Two Japanese patients with heterozygous R121W mutation in PAX4 were treated with diet alone, similar to the father in our study (Shimajiri et al. 2001). It may be speculated that the father had decreased insulin secretory response or insulin resistance, which improved after diet therapy. In contrast, the proband has continued to require insulin therapy. Shimajiri et al. (2001) also reported that two other patients with $\mathrm{R} 121 \mathrm{~W}$ mutation were treated with insulin transiently. Therefore, there is still a possibility that insulin may be able to be withdrawn from the proband and thus periodic evaluation of his insulin secretion capacity must be performed.

Similar to our familial case, in Thai patients, the phe- notype varied in the same family (Plengvidhya et al. 2007). The detailed clinical history of this patient with IVS7$1 \mathrm{G}>\mathrm{A}$ mutation was not described in the literature; however, diabetes in this pedigree seems to be severe and was characterized by early onset of renal complications. These variable phenotypes may thus be accounted for by the implication of other genetic and/or environmental factors (Peltonen and Mckusick 2001).

In conclusion, we identified a novel mutation of PAX4, which might be pathogenic. In addition, this family provides another example that there may be phenotypic heterogeneity within a MODY family. 


\section{Acknowledgments}

We thank Dr. Furuta and Dr. Nanjo (First Department of Medicine, Wakayama University of Medical Science, Wakayama, Japan) for providing us with PAX4 expression vector and insulin and glucagon promoter vectors for the luciferase assay.

\section{References}

Blencowe, B.J. (2000) Exonic splicing enhancers: mechanism of action, diversity and role in human genetic diseases. Trends Biochem. Sci., 25, 106-110.

Brun, T. \& Gauthier, B.R. (2008) A focus on the role of Pax4 in mature pancreatic islet beta-cell expansion and survival in health and disease. J. Mol. Endocrinol., 40, 37-45.

Fajans, S.S., Bell, G.I. \& Polonsky, K.S. (2001) Molecular mechanisms and clinical pathophysiology of maturity-onset diabetes of the young. N. Engl. J. Med., 345, 971-980.

Fujitani, Y., Kajimoto, Y., Yasuda, T., Matsuoka, T.A., Kaneto, H., Umayahara, Y., Fujita, N., Watada, H., Miyazaki, J.I., Yamasaki, Y. \& Hori, M. (1999) Identification of a portable repression domain and an E1A-responsive activation domain in Pax4: a possible role of Pax4 as a transcriptional repressor in the pancreas. Mol. Cell. Biol., 19, 8281-8291.

Furuta, H., Furuta, M., Sanke, T., Ekawa, K., Hanabusa, T., Nishi, M., Sasaki, H. \& Nanjo, K. (2002) Nonsense and missense mutations in the human hepatocyte nuclear factor-1 beta gene (TCF2) and their relation to type 2 diabetes in Japanese. $J$. Clin. Endocrinol. Metab., 87, 3859-3863.

Horikawa, Y., Iwasaki, N., Hara, M., Furuta, H., Hinokio, Y., Cockburn, B.N., Lindner, T., Yamagata, K., Ogata, M., Tomonaga, O., Kuroki, H., Kasahara, T., Iwamoto, Y. \& Bell, G.I. (1997) Mutation in hepatocyte nuclear factor-1 beta gene (TCF2) associated with MODY. Nat. Genet., 17, 384-385.

Kagami-Takasugi, M., Katsumata, N., Tanaka, T., Tajima, T. \& Fujieda, K. (2006) Molecular genetic analysis of MODY candidate genes in Japanese patients with non-obese juvenile onset diabetes mellitus. J. Pediatr. Endocrinol. Metab., 19, 143-148.

Levit, A., Nutman, D., Osher, E., Kamhi, E. \& Navon, R. (2010) Two novel exonic point mutations in HEXA identified in a juvenile Tay-Sachs patient: role of alternative splicing and nonsense-mediated mRNA decay. Mol. Genet. Metab., 100, 176-183.

Malecki, M.T., Jhala, U.S., Antonellis, A., Fields, L., Doria, A., Orban, T., Saad, M., Warram, J.H., Montminy, M. \& Krolewski, A.S. (1992) Mutations in NEUROD1 are associated with the development of type 2 diabetes mellitus. Nat. Genet., 23, 323-328.

Moseley, C.T., Mullis, P.E., Prince, M.A. \& Phillips, J.A. 3rd. (2002) An exon splice enhancer mutation causes autosomal dominant GH deficiency. J. Clin. Endocrinol. Metab., 87, 847-852.

Nishigori, H., Yamada, S., Kohama, T., Utsugi, T., Shimizu, H., Takeuchi, T. \& Takeda, J. (1993) Mutations in the hepatocyte nuclear factor-1 alpha gene (MODY3) are not a major cause of early-onset non-insulin-dependent (type 2) diabetes mellitus in Japanese. J. Hum. Genet., 43, 107-110.

Peltonen, L. \& McKusick, V.A. (2001) Genomics and medicine. Dissecting human disease in the postgenomic era. Science, 291, 1224-1229.

Plengvidhya, N., Kooptiwut, S., Songtawee, N., Doi, A., Furuta, H., Nishi, M., Nanjo, K., Tantibhedhyangkul, W., Boonyasrisawat, W., Yenchitsomanus, P.T., Doria, A. \& Banchuin, N. (2007) PAX4 mutations in Thais with maturity onset diabetes of the young. J. Clin. Endocrinol. Metab., 92, 2821-2826.

Rehwinkel, J., Raes, J. \& Izaurralde, E. (2006) Nonsense-mediated mRNA decay: target genes and functional diversification of effectors. Trends Biochem. Sci., 31, 639-646.

Seino, Y., Nanjo, K., Tajima, N., Kadowaki, T., Kashiwagi, A., Araki, E., Ito, C., Inagaki, N., Iwamoto, Y., Kasuga, M., Hanafusa, T., Haneda, M. \& Ueki, K. (2010) Report of the Committee in the classification and diagnostic criteria of diabetes mellitus. J. Diabetes Invest., 1, 212-228.

Shimajiri, Y., Sanke, T., Furuta, H., Hanabusa, T., Nakagawa, T., Fujitani, Y., Kajimoto, Y., Takasu, N. \& Nanjo, K. (2001) A missense mutation of Pax4 gene (R121W) is associated with type 2 diabetes in Japanese. Diabetes, 50, 2864-2869.

Smith, S.B., Ee, H.C., Conners, J.R. \& German, M.S. (1999) Paired-homeodomain transcription factor PAX4 acts as a transcriptional repressor in early pancreatic development. Mol. Cell. Biol., 9, 8272-8280.

Sosa-Pineda, B., Chowdhury, K., Torres, M., Oliver, G. \& Gruss, P. (1997) The Pax4 gene is essential for differentiation of insulin-producing beta cells in the mammalian pancreas. Nature, 386, 399-402.

Sosa-Pineda, B. (2004) The gene Pax4 is an essential regulator of pancreatic beta-cell development. Mol. Cells, 18, 289-294.

Stoffers, D.A., Ferrer, J., Clarke, W.L. \& Habener, J.F. (1997) Early-onset type-II diabetes mellitus (MODY4) linked to IPF1. Nat. Genet., 17, 138-139.

Vionnet, N., Stoffel, M., Takeda, J., Yasuda, K., Bell, G.I., Zouali, H., Lesage, S., Velho, G., Iris, F., Passa, P., Froguel, P. \& Cohen, D. (1992) Nonsense mutation in the glucokinase gene causes early-onset non-insulin-dependent diabetes mellitus. Nature, 356, 721-722.

Yamagata, K., Furuta, H., Oda, N., Kaisaki, P.J., Menzel, S., Cox, N.J., Fajans, S.S., Signorini, S., Stoffel, M. \& Bell, G.I. (1996a) Mutations in the hepatocyte nuclear factor-4alpha gene in maturity-onset diabetes of the young (MODY1). Nature, 384, 458-460.

Yamagata, K., Oda, N., Kaisaki, P.J., Menzel, S., Furuta, H., Vaxillaire, M., Southam, L., Cox, R.D., Lathrop, M.G., Boriraj, V.V., Chen, X., Cox, N.J., Oda, Y., Yano, H., LeBeau, M.M., Yamada, S., Nishigori, H., Takeda, J., Fajans, S.S., Hattersley, A.T., Iwasaki, N., Hansen, T., Pederson, O., Polonsky, K.S., Turner, R.C., Velho, G., Chevre J.C., Froguel, P. \& Bell, G.I. (1996b) Mutations in the hepatocyte nuclear factor-1alpha gene in maturity-onset diabetes of the young (MODY3). Nature, 384, 455-458. 